\title{
Fatores associados ao consumo de frutas e verduras entre adolescentes
}

\section{Factors associated with the consumption of fruits and vegetables among adolescents}

\author{
Raquel Raizela, Allan da Mata Godois ${ }^{b}$, Valdemar Guedes da Silvac, \\ Christianne de Faria Coelho Ravagnanid \\ Nutricionista. Doutora em Ciências dos Alimentos. Departamento de Alimentos e Nutrição Experimental, FCF - Universidade de São Paulo. \\ b Nutricionista e Educador Físico. Mestre em Biociências-Nutrição. Faculdade de Nutrição, FANUT - Universidade Federal de Mato Grosso. \\ cEducador Físico. Mestre em Educação Física. Faculdade de Educação Física, FEF - Universidade Federal de Mato Grosso. \\ dNutricionista e Educadora Física. Pós-Doutorado em Exercise Science. Faculdade de Educação Física - Universidade Federal de Mato Grosso do Sul. \\ Fontes de financiamento: Bolsa de estudos CAPES.
}

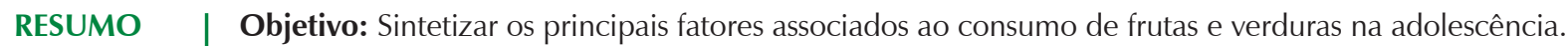

Materiais e Métodos: Foi realizada uma busca nas bases: LILACS, SciELO, ADOLEC, PubMed, e Cochrane library. Os termos frutas, verduras e hortaliças foram retirados do Medical Subject Heading e Descritores em Ciências da Saúde e, utilizados nos idiomas: Português, Espanhol e Inglês. Foram incluídos 39 estudos com adolescentes de 10 a 19 anos, cujo desfecho foi o consumo de frutas e/ou verduras.

Resultados: Aspectos sociodemográficos, alimentação familiar e apoio dos colegas estão associados à maior ingestão de frutas e verduras. Entretanto, beber, fumar, atividades sedentárias, inatividade física, insegurança alimentar, acesso a alimentos não saudáveis, influência midiática e moradia em região urbana estão inversamente associados ao consumo destes alimentos.

Conclusão: Fatores sociodemográficos, estilo de vida, a influência da família e dos amigos representam os principais fatores associados à ingestão de frutas e verduras na adolescência e, estes, deveriam ser integrados a programas de promoção/educação à saúde para aumentar o consumo diário de frutas e verduras entre adolescentes.

Palavras-chave: frutas; verduras; adolescentes.

\begin{tabular}{l|l} 
ABSTRACT & Objective: To summarize the main factors associated with fruit and vegetable consumption in adolescence.
\end{tabular}

Materials and Methods: A search was performed on LILACS, SCIELO, ADOLEC, PubMed, and the Cochrane library. The terms fruit and vegetables were taken from the Medical Subject Heading and Health Sciences Descriptors, in Portuguese, Spanish and English. A total of 39 articles with adolescents aged 10 to 19 years old, whose outcome was fruit and/or vegetables consumption were included. Results: Sociodemographic factors, family diet and peer support are associated with higher consumption of fruit and vegetables. However, drinking, smoking, sedentary activities, physical inactivity, food insecurity, access to unhealthy foods, media influence and housing in urban areas are inversely associated.

Conclusion: Sociodemographic factors, lifestyle and the influence of family and friends are the main factors associated with fruit and vegetables consumption in adolescence and it should be integrated to health education/promotion programs to increase daily fruit and vegetable consumption among adolescents.

Keywords: fruit; vegetables; adolescents.

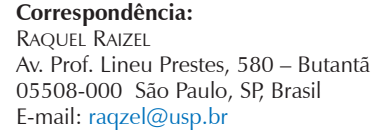




\section{INTRODUÇÃO}

As doenças crônicas não transmissíveis representam um importante desafio de saúde pública devido ao risco de morbidade e mortalidade, e a alimentação não saudável e o excesso de peso estão entre os principais fatores de risco para estas doenças ${ }^{1}$. Pesquisas sugerem que o aumento da ingestão de frutas e verduras poderia prevenir este cenário, contribuindo na redução da adiposidade corporal $^{2}$, do risco de mortalidade por doenças não transmissíveis ${ }^{3-5}$, da prevalência de câncer gástrico ${ }^{3,6}$ e outros tipos de cânceres em adultos ${ }^{3,7,8}$. Além disso, a dieta rica em frutas e verduras está inversamente associada à pressão sanguínea diastólica ${ }^{9} \mathrm{e}$ ao risco de sobrepeso e obesidade na adolescência ${ }^{10,11}$. Considerando que o comportamento alimentar adquirido na adolescência parece permanecer na vida adulta ${ }^{12}$, a mudança de hábitos durante a adolescência poderia prevenir algumas enfermidades. Contudo, apesar dos prejuízos ocasionados por alimentação inadequada, o consumo de frutas e verduras entre adolescentes ainda está aquém da recomendação diária de $400 \mathrm{~g}^{13,14}$.

Políticas mundiais de saúde pública são implementadas com o propósito de incentivar o aumento da ingestão destes alimentos ${ }^{15,16}$. Mas há de se considerar os aspectos culturais, sociais, afetivos e sensoriais envolvidos na alimentação e, para que as políticas e programas de intervenção na adolescência sejam realmente eficientes, é importante identificar previamente os fatores associados ao comportamento alimentar, ou seja, aqueles sobre os quais se devem intervir. Em busca da compreensão destes fatores, alguns autores identificaram que o incentivo familiar, a ingestão dietética dos pais, as regras alimentares impostas pela família e, a disponibilidade ou acessibilidade dos alimentos, estão positivamente associadas ao consumo de frutas e verduras de adolescentes ${ }^{17,18}$. Adicionalmente, a forma de preparo, aspectos sensoriais e físicos, o sabor e variedade são fatores associados a uma dieta rica em frutas e verduras ${ }^{19}$.

Os fatores associados ao consumo de frutas e verduras de adolescentes podem estar inseridos em múltiplas dimensões. Contudo, as investigações estão centradas majoritariamente no ambiente familiar ${ }^{18}$, sociocultural e econômico ${ }^{17}$. O que não parece ser apropriado, pois desconsidera outros fatores do contexto sob o qual o indivíduo está inserido, como aspectos individuais e os dificilmente atingíveis como influência midiática. Neste sentido, considerando a relevância da temática para a saúde pública, o objetivo do presente trabalho foi revisar e sintetizar os principais fatores associados ao consumo de frutas e verduras de adolescentes e, com isso, sugerir ações de intervenção sobre estes fatores.

\section{MATERIAIS E MÉTODOS}

\section{Estratégia de busca}

Foi realizada uma busca de estudos indexados nas bases: LILACS, SCIELO, ADOLEC (área especializada em adolescentes) e PubMed. Adicionalmente, foram checadas referências de revisões sistemáticas registradas na Cochrane library. Os termos foram retirados do MeSH (Medical Subject Heading) e DeCS (Descritores em Ciências da Saúde), nos idiomas Português, Espanhol e Inglês. A busca foi realizada sem restrição de período, por meio dos formulários avançados nas bases LILACS, SCIELO e ADOLEC.

Foram utilizadas as combinações entre os termos em português: frutas, verduras e hortaliças, e os respectivos termos no idioma Espanhol, nas bases LILACS, SciELO e ADOLEC. Para refinamento da busca na base de dados PubMed, foram aplicados os limites: humans (estudos realizados com humanos); Adolescent; e restrição de idiomas (English, Portuguese, Spanish):

1. ("fruit" [MeSH Terms] OR "fruit" [All Fields]) AND ("humans" [MeSH Terms] AND (English [lang] OR Portuguese [lang] OR Spanish [lang]) AND "adolescent" [MeSH Terms]);

2. ("vegetables" [MeSH Terms] OR "vegetables" [All Fields]) AND ("humans" [MeSH Terms] AND (English [lang] OR Portuguese [lang] OR Spanish [lang]) AND "adolescent" [MeSH Terms]).

As etapas da revisão foram divididas em: i) triagem dos títulos ii) triagem dos resumos e iii) leitura integral do artigo. Foram considerados elegíveis os artigos: 1) originais, com delineamento transversal e/ ou coorte; 2) com adolescentes sendo sujeitos do estudo, com idade entre 10 e 19 anos (ou média neste intervalo); 3) consumo de frutas, verduras ou hortaliças como variável dependente; 4) que examinaram pelo menos um fator de associação ao consumo de frutas e verduras; 5) publicados em Inglês, Espanhol ou Português.

Foram excluídos estudos: 1) com foco em distúrbios alimentares; 2) que avaliaram o consumo alimentar em apenas uma refeição/dia; 3) quando os dados dos adolescentes não pudessem ser extraídos a partir dos dados de outros grupos etários; 4) com amostragem por conveniência ou sem descrição do processo de seleção dos participantes; 5) de intervenção; 6) apenas com adolescentes acima do peso ou obesos; 7) não publicados em revistas científicas (como resumos, dissertações ou teses); 8) publicados em outros idiomas.

\section{Extração de dados}

A extração de dados foi conduzida independentemente por dois revisores (RR e AMG) com formulário padronizado 
desenvolvido para este estudo. O formulário continha as informações: autor, ano de publicação, país de coleta, delineamento, tamanho da amostra, idade, sexo, desfecho, instrumento de coleta de dados, descrição da forma de utilização e validação do instrumento, indicação de confiabilidade das medidas, variáveis independentes, análise estatística, resultados da análise, descrição das limitações do estudo e taxa de respostas. Os dados extraídos foram comparados e distribuídos em tabelas.

\section{Análise dos dados extraídos}

As associações foram consideradas significativas quando $p<0,05$, e agrupadas em: positivas, inversas, e sem associação significativa. Para estudos com resultados de análise univariada, bivariada e multivariada, somente os resultados da análise multivariada foram incluídos. Foi possível verificar estudos com mais de uma amostra, porém com resultados provenientes da combinação das amostras para análise. Outros com descrição do tamanho amostral total, entretanto com análise estratificada por idade, dessa forma, foram apresentados somente os resultados para adolescentes.

Por compreender que o gênero pode influenciar o comportamento alimentar, foram apresentados resultados de análises estratificadas por sexo e considerados os dados de estudos longitudinais correspondentes a adolescentes. As associações entre consumo alimentar e diferentes nacionalidades foram excluídas. Para reduzir o número de variáveis independentes, fatores com características similares foram combinados examinando-se a descrição na publicação de origem (como exemplo a frequência de realização do jantar em família uniu-se a realizar o jantar diariamente com os pais). Os fatores foram agrupados quando relacionados ao próprio indivíduo, a família, parentes e amigos.

\section{RESULTADOS}

Foram localizados 21.422 artigos e, após revisão, 39 foram incluídos neste estudo (Figura 1). As características dos estudos foram mostradas na Tabela 1. Os estudos selecionados foram conduzidos majoritariamente nos Estados Unidos $(n=14)$, seguido pelo Brasil $(n=6)$. A maioria com delineamento transversal $(n=35)$, e tamanho amostral de 145 a 162.305. A ingestão inadequada de frutas e verduras foi examinada como desfecho em oito estudos e a maioria avaliou fatores relacionados ao consumo adequado $(n=31)$. Destes, três examinaram apenas o consumo de frutas como variável dependente e dois outros, o consumo de verduras. As análises foram estratificadas por sexo em doze artigos, e vinte e sete examinaram fatores associados ao consumo alimentar sem diferenciar o gênero. O questionário de frequência alimentar (QFA) foi o instrumento de avaliação mais utilizado $(n=19)$ e apenas um estudo ${ }^{40}$ utilizou relato dos pais para identificação da alimentação de menores de 15 anos. Boa parte dos estudos relatou validade do instrumento $(n=18)$ e confiabilidade nas medições do consumo alimentar $(n=21)$.

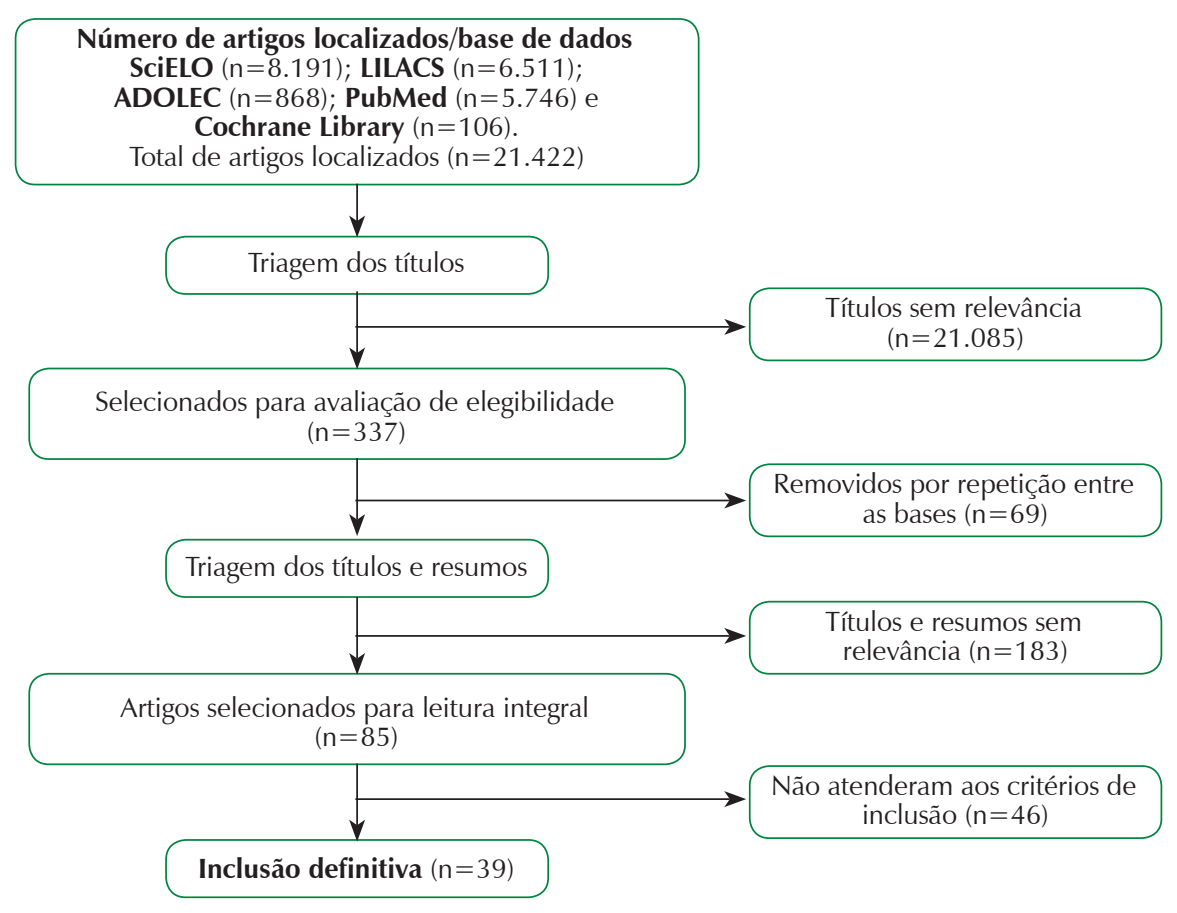

Figura 1. Fluxograma de seleção dos estudos. 
Tabela 1. Características gerais dos estudos com adolescentes de 10 a 19 anos.

\begin{tabular}{|c|c|}
\hline Características & Referências \\
\hline \multicolumn{2}{|l|}{ Tamanho amostral } \\
\hline$<500$ & $\begin{array}{l}\text { [Young et al., 2004; Monge-Rojas et al., 2010H/M; Fulkerson et al., 2009; Assumpção et al., } \\
\text { 2012H/M] }\end{array}$ \\
\hline $500-699$ & $\begin{array}{l}\text { [Bigio et al., 2011; Beydoun et al., 2011; Martens et al., 2005; Arcan et al., 2007H/M; Burgess- } \\
\text { Champoux et al., 2009H/M, Hanson et al., 2005H/M; Lien et al., 2002H/M; Moraes et al., } \\
\text { 2010H/M; Moraes et al., 2012H/M; Kremers et al., 2003; Monge-Rojas et al., 2002; Giskes et al., } \\
\text { 2002; Lachat et al., 2009H/M; Shi et al., 2005H/M; Gerrits et al., 2010; Fernandes et al., 2011] }\end{array}$ \\
\hline 2000-7999 & $\begin{array}{l}\text { [Bruening et al., 2012; Kourlaba et al., 2009; Cutler et al., 2011; Ball et al., 2009; Freisling et al., } \\
\text { 2010; Pedersen et al., 2012H/M; Shi et al., 2010; Neumark-Sztainer et al., 2003; Farias et al., 2009; } \\
\text { Stewart \& Menning, 2009l/II; Ortiz-Hernández \& Gómez-Tello, 2008] }\end{array}$ \\
\hline$>7999$ & $\begin{array}{l}\text { [Verzeletti et al., 2010; Peltzer \& Pengpid, 2012; Peltzer \& Pengpid, 2010; Videon et al., 2003; } \\
\text { Riediger et al., 2007; Neumark-Sztainer et al., 1996; Roos et al., 2001H/M; Vereecken et al., 2006] }\end{array}$ \\
\hline \multicolumn{2}{|l|}{ Gênero } \\
\hline Homens ou mulheres & $\begin{array}{l}\text { [Arcan et al., 2007; Assumpção et al., 2012; Burgess-Champoux et al., 2009; Hanson et al., 2005; } \\
\text { Lachat et al., 2009; Lien et al., 2002; Monge-Rojas et al., 2010; Moraes et al., 2010; Moraes et al., } \\
\text { 2012; Pedersen et al., 2012; Shi et al., 2005; Roos et al., 2001] }\end{array}$ \\
\hline Homens e mulheres & $\begin{array}{l}\text { [Beydoun et al., 2011; Ortiz-Hernández \& Gómez-Tello, 2008; Martens et al., 2005; Riediger et al., } \\
\text { 2007; Verzeletti et al., 2010; Young et al., 2004; Vereecken et al., 2006; Freisling et al., 2010; } \\
\text { Peltzer \& Pengpid, 2010; Peltzer \& Pengpid, 2012; Ball et al., 2009; Neumark-Sztainer et al., 1996; } \\
\text { Shi et al., 2010; Bruening et al., 2012; Monge-Rojas et al., 2002; Cutler et al., 2011; Giskes et al., } \\
\text { 2002; Videon et al., 2003; Farias et al., 2009; Fulkerson et al., 2009; Gerrits et al., 2010; Kremers et al., } \\
\text { 2003; Bigio et al., 2011; Fernandes et al., 2011; Kourlaba et al., 2009; Neumark-Sztainer et al., 2003; } \\
\text { Stewart \& Menning, 2009l/II] }\end{array}$ \\
\hline \multicolumn{2}{|l|}{ Delineamento } \\
\hline Longitudinal & $\begin{array}{l}\text { [Arcan et al., 2007H/M; Burgess-Champoux et al., 2009H/M; Stewart \& Menning, 2009I/II, Lien et al., } \\
2002 \mathrm{H} / \mathrm{M} \text { ] }\end{array}$ \\
\hline Transversal & $\begin{array}{l}\text { [Beydoun et al., 2011; Ortiz-Hernández \& Gómez-Tello, 2008; Martens et al., 2005; Riediger et al., } \\
\text { 2007; Verzeletti et al., 2010; Young et al., 2004; Cutler et al., 2011; Hanson et al., 2005H/M; } \\
\text { Vereecken et al., 2006; Freisling et al., 2010; Peltzer \& Pengpid, 2010; Peltzer \& Pengpid, 2012; } \\
\text { Ball et al., 2009; Roos et al., 2001H/M; Neumark-Sztainer et al., 1996; Pedersen et al., 2012H/M; } \\
\text { Monge-Rojas et al., 2010H/M; Shi et al., 2010; Bruening et al., 2012; Monge-Rojas et al., 2002; } \\
\text { Giskes et al., 2002; Videon et al., 2003; Farias et al., 2009; Lachat et al., 2009H/M, Shi et al., } \\
\text { 2005H/M; Fulkerson et al., 2009; Assumpção et al., 2012H/M; Gerrits et al., 2010; Kremers et al., } \\
\text { 2003; Bigio et al.., 2011; Moraes et al., 2012H/M; Fernandes et al., 2011; Moraes et al., 2010H/M; } \\
\text { Kourlaba et al., 2009; Neumark-Sztainer et al.,, 2003] }\end{array}$ \\
\hline
\end{tabular}

Desfecho dietético

Frutas

Verduras

Frutas e verduras

Frutas/suco/ verduras

Ingestão inadequada de frutas e verduras

\footnotetext{
Meio de avaliação

Relato (adolescente/pais)

Auto relato (adolescente)
}

\section{[Ball et al., 2009; Kremers et al., 2003; Martens et al., 2005]}

[Stewart \& Menning, 2009I/II, Roos et al., 2001H/M]

[Beydoun et al., 2011; Bigio et al., 2011; Cutler et al., 2011; Gerrits et al., 2010; Lachat et al., 2009H/M, Lien et al., 2002H/M; Monge-Rojas et al., 2002; Moraes et al., 2012H/M; Shi et al., 2010; Riediger et al., 2007; Young et al., 2004]

[Arcan et al., 2007H/M; Hanson et al., 2005H/M; Giskes et al., 2002; Kourlaba et al., 2009; Shi et al., 2005H/M; Bruening et al., 2012; Burgess-Champoux et al., 2009H/M; Fernandes et al., 2011; Freisling et al., 2010; Fulkerson et al., 2009; Ortiz-Hernández \& Gómez-Tello, 2008; Monge-Rojas et al., 2010H/M; Vereecken et al., 2006; Neumark-Sztainer et al., 2003; Verzeletti et al., 2010]

[Peltzer \& Pengpid, 2010; Assumpção et al., 2012H/M; Farias et al., 2009; Pedersen et al., 2012H/M; Peltzer \& Pengpid, 2012; Videon et al., 2003; Neumark-Sztainer et al., 1996; Moraes et al., $2010 \mathrm{H} / \mathrm{M}]$

[Giskes et al., 2002]

[Burgess-Champoux et al., 2009H/M; Beydoun et al., 2011; Ortiz-Hernández \& Gómez-Tello, 2008; Martens et al., 2005; Riediger et al., 2007; Verzeletti et al., 2010; Young et al., 2004; Arcan et al., 2007H/M; Hanson et al., 2005H/M; Vereecken et al., 2006; Freisling et al., 2010; Peltzer \& Pengpid, 2010; Peltzer \& Pengpid, 2012; Ball et al., 2009; Roos et al., 2001H/M; Neumark-Sztainer et al., 1996; Pedersen et al., 2012HM; Monge-Rojas et al., 2010H/M; Shi et al., 2010; Bruening et al., 2012; Monge-Rojas et al., 2002; Lien et al., 2002H/M; Cutler et al., 2011; Videon et al., 2003; Farias et al., 2009; Lachat et al., 2009H/M; Shi et al., 2005H/M; Fulkerson et al., 2009; Assumpção et al., 2012H/M; Gerrits et al., 2010; Kremers et al., 2003; Bigio et al., 2011; Moraes et al., 2012H/M; Fernandes et al., 2011; Moraes et al., 2010H/M; Kourlaba et al., 2009; Neumark-Sztainer et al., 2003; Stewart \& Menning, 2009I/II] 
Tabela 1 (continuação)

\begin{tabular}{|c|c|}
\hline Características & Referências \\
\hline \multicolumn{2}{|l|}{ Instrumento } \\
\hline QFA & $\begin{array}{l}\text { [Arcan et al., 2007H/M; Vereecken et al., 2006; Ball et al., 2009; Neumark-Sztainer et al., 1996; } \\
\text { Bruening et al., 2012; Burgess-Champoux et al., 2009H/M; Cutler et al., 2011; Stewart \& Menning, } \\
\text { 2009l/II, Freisling et al., 2010; Hanson et al., 2005H/M; Kourlaba et al., 2009; Kremers et al., 2003; } \\
\text { Martens et al., 2005; Moraes et al., 2010H/M; Moraes et al., 2012H/M; Pedersen et al., 2012H/M; } \\
\text { Shi et al., 2005H/M; Roos et al., 2001H/M; Neumark-Sztainer et al., 2003] }\end{array}$ \\
\hline Questionário & $\begin{array}{l}\text { [Farias et al., 2009; Fernandes et al., 2011; Fulkerson et al., 2009; Ortiz-Hernández \& Gómez-Tello, } \\
\text { 2008; Lien et al., 2002H/M; Monge-Rojas et al., 2002; Peltzer \& Pengpid, 2010; Peltzer \& Pengpid, } \\
\text { 2012; Riediger et al., 2007; Young et al., 2004] }\end{array}$ \\
\hline Entrevista & [Gerrits et al., 2010; Shi et al., 2010; Verzeletti et al., 2010; Videon et al., 2003] \\
\hline Rec. $24 \mathrm{~h}$ & $\begin{array}{l}\text { [Assumpção et al., 2012H/M; Beydoun et al., 2011; Bigio et al., 2011; Giskes et al., 2002; } \\
\text { Lachat et al., 2009H/M] }\end{array}$ \\
\hline Registro (3 dias) & [Monge-Rojas et al., 2010H/M] \\
\hline \multicolumn{2}{|l|}{ Confiabilidade } \\
\hline Relatada & $\begin{array}{l}\text { [Arcan et al., 2007H/M; Bigio et al., 2011; Bruening et al., 2012; Burgess-Champoux et al., } \\
2009 H / M \text {; Cutler et al., 2011; Gerrits et al., 2010; Giskes et al., 2002; Hanson et al., 2005H/M; } \\
\text { Lachat et al., 2009H/M; Lien et al., 2002H/M; Monge-Rojas et al., 2010H/M; Monge-Rojas et al., } \\
\text { 2002; Moraes et al., 2010H/M; Moraes et al., 2012H/M; Vereecken et al., 2006; Shi et al., } \\
\text { 2005H/M; Neumark-Sztainer et al., 1996; Neumark-Sztainer et al., 2003; Riediger et al., 2007; } \\
\text { Verzeletti et al., 2010; Young et al., 2004] }\end{array}$ \\
\hline Não relatada & $\begin{array}{l}\text { [Assumpção et al., 2012H/M; Ball et al., 2009; Beydoun et al., 2011; Farias et al., 2009; } \\
\text { Stewart \& Menning, 2009I/II, Fernandes et al., 2011; Freisling et al., 2010; Fulkerson et al., 2009; } \\
\text { Ortiz-Hernández \& Gómez-Tello, 2008; Kourlaba et al., 2009; Kremers et al., 2003; Martens et al., } \\
\text { 2005; Pedersen et al., 2012H/M; Shi et al., 2010; Roos et al., 2001H/M; Peltzer \& Pengpid, 2010; } \\
\text { Peltzer \& Pengpid, 2012; Videon et al., 2003] }\end{array}$ \\
\hline \multicolumn{2}{|l|}{ Validade da medição } \\
\hline Relatada & $\begin{array}{l}\text { [Arcan et al., 2007H/M; Ball et al., 2009; Farias et al., 2009; Bruening et al., 2012; Burgess- } \\
\text { Champoux et al., 2009H/M; Cutler et al., 2011; Fulkerson et al., 2009; Hanson et al., 2005H/M; } \\
\text { Kremers et al., 2003; Lien et al., 2002H/M; Martens et al., 2005; Monge-Rojas et al., 2010H/M; } \\
\text { Monge-Rojas et al., 2002; Moraes et al., 2010H/M; Moraes et al., 2012H/M; Vereecken et al., 2006; } \\
\text { Neumark-Sztainer et al., 1996; Verzeletti et al., 2010] }\end{array}$ \\
\hline Não relatada & $\begin{array}{l}\text { [Beydoun et al., 2011; Ortiz-Hernández \& Gómez-Tello, 2008; Riediger et al., 2007; Young et al., 2004; } \\
\text { Freisling et al., 2010; Peltzer \& Pengpid, 2010; Peltzer \& Pengpid, 2012; Roos et al., 2001H/M; } \\
\text { Pedersen et al., 2012H/M; Shi et al., 2010; Giskes et al., 2002; Videon et al., 2003; Lachat et al., } \\
\text { 2009H/M; Shi et al.,, 2005H/M; Assumpçâo et al., 2012H/M; Gerrits et al., 2010; Bigio et al., 2011; } \\
\text { Fernandes et al., 2011; Kourlaba et al.,, 2009; Neumark-Sztainer et al., 2003; Stewart \& Menning, 2009l/II] }\end{array}$ \\
\hline \multicolumn{2}{|l|}{ Análise dos dados } \\
\hline Bivariada & [Arcan et al., 2007H/M] \\
\hline Multivariada & $\begin{array}{l}\text { [Bigio et al., 2011; Bruening et al., 2012; Burgess-Champoux et al., 2009H/M; Cutler et al., 2011; } \\
\text { Stewart \& Menning, 2009l//I, Freisling et al., 2010; Fulkerson et al., 2009; Giskes et al., 2002; } \\
\text { Ortiz-Hernández \& Gómez-Tello, 2008; Martens et al., 2005] }\end{array}$ \\
\hline Uni, Bi/Multivariada & $\begin{array}{l}\text { [Beydoun et al., 2011; Ortiz-Hernández \& Gómez-Tello, 2008; Martens et al., 2005; Riediger et al., } \\
\text { 2007; Verzeletti et al., 2010; Young et al., 2004; Hanson et al., 2005H/M; Vereecken et al., 2006; } \\
\text { Shi et al., 2010; Peltzer \& Pengpid, 2010; Peltzer \& Pengpid, 2012; Ball et al., 2009; Roos et al., } \\
\text { 2001H/M; Neumark-Sztainer et al., 1996; Pedersen et al., 2012H/M; Videon et al., 2003; } \\
\text { Monge-Rojas et al., 2010H/M; Monge-Rojas et al., 2002; Lien et al., 2002H/M; Farias et al., 2009; } \\
\text { Lachat et al., 2009H/M; Shi et al., 2005H/M; Assumpção et al., 2012H/M; Gerrits et al.,, 2010; } \\
\text { Kremers et al., 2003; Moraes et al., 2012H/M; Fernandes et al., 2011; Moraes et al., 2010H/M; } \\
\text { Kourlaba et al., 2009; Neumark-Sztainer et al., 2003] }\end{array}$ \\
\hline \multicolumn{2}{|l|}{ País de estudo } \\
\hline Estados Unidos (US) & $\begin{array}{l}\text { [Arcan et al., 2007H/M; Bruening et al., 2012; Burgess-Champoux et al., 2009H/M; Cutler et al., 2011; } \\
\text { Stewart \& Menning, 2009I/II, Fulkerson et al., 2009; Hanson et al., 2005H/M; Lien et al., 2002H/M; } \\
\text { Shi et al., 2010; Neumark-Sztainer et al., 1996; Neumark-Sztainer et al., 2003; Videon et al., 2003] }\end{array}$ \\
\hline Brasil & $\begin{array}{l}\text { [Assumpção et al., 2012H/M; Bigio et al., 2011; Farias et al., 2009; Fernandes et al., 2011; } \\
\text { Moraes et al., 2010H/M; Moraes et al., 2012H/M] }\end{array}$ \\
\hline Países europeus & $\begin{array}{l}\text { [Ball et al., 2009; Beydoun et al., 2011; Freisling et al., 2010; Giskes et al., 2002; Kourlaba et al., } \\
\text { 2009; Kremers et al., 2003; Martens et al., 2005; Pedersen et al., 2012H/M; Roos et al., 2001H/M; } \\
\text { Verzeletti et al., 2010; Young et al., 2004] }\end{array}$ \\
\hline Países asiáticos & [Lachat et al., 2009H/M; Shi et al., 2005H/M; Peltzer \& Pengpid, 2012] \\
\hline Países americanos & $\begin{array}{l}\text { [Riediger et al., 2007; Ortiz-Hernández \& Gómez-Tello, 2008; Monge-Rojas et al., 2002; } \\
\text { Monge-Rojas et al., 2010H/M] }\end{array}$ \\
\hline Europa, US, Israel, Canadá, África, Hungria & [Vereecken et al., 2006; Peltzer \& Pengpid, 2010; Gerrits et al., 2010] \\
\hline
\end{tabular}

H/M: Homens e Mulheres analisados separadamente; //II: amostras independentes; QFA: Questionário de Frequência Alimentar; Rec. 24h: Recordatório de 24 horas; Uni: Univariada; Bi: Bivariada. 
Foram encontrados 87 fatores relacionados ao consumo de frutas e verduras de adolescentes, os quais foram agrupados em nove temas: comportamentos de risco e de saúde; peso corporal e percepção dos adolescentes; hábito alimentar; meio de acesso às informações sobre alimentação; influência familiar; influência de amigos e colegas; disponibilidade e acessibilidade aos alimentos; fatores socioeconômicos; fatores demográficos. Os temas foram descritos e os fatores sintetizados nas Tabelas 2, 3 e 4 .

Tabela 2. Fatores associados ao consumo de frutas de adolescentes (10 a 19 anos).

\begin{tabular}{|c|c|c|c|}
\hline \multirow{2}{*}{ Fatores estudados } & \multicolumn{3}{|c|}{ Referências } \\
\hline & Associações positivas & Associações inversas & Sem associação \\
\hline \multicolumn{4}{|l|}{ Comportamentos de risco e de saúde } \\
\hline Uso de tabaco & & $\begin{array}{l}\text { [Freisling et al., 2010; Peltzer \& } \\
\text { Pengpid, 2010] }\end{array}$ & $\begin{array}{l}\text { [Kourlaba et al., 2009; Lien et al., } \\
\text { 2002; Peltzer \& Pengpid, 2012] }\end{array}$ \\
\hline Comportamento sedentário & & $\begin{array}{l}\text { [Vereecken et al., 2006; Freisling } \\
\text { et al., 2010; Verzeletti et al., 2010; } \\
\text { Peltzer \& Pengpid, 2010] }\end{array}$ & [Fernandes et al., 2011] \\
\hline Ser ativo & [Fernandes et al., 2011] & & \\
\hline \multicolumn{4}{|l|}{ Peso corporal e Percepções } \\
\hline IMC elevado & [Assumpção et al., 2012] & & $\begin{array}{l}\text { [Bigio et al., 2011; Freisling et al., } \\
\text { 2010; Gerrits et al., 2010] }\end{array}$ \\
\hline Estar acima do peso & & [Neumark-Sztainer et al., 1996] & \\
\hline $\begin{array}{l}\text { Atitude/Habilidade para escolher } \\
\text { alimentos saudáveis }\end{array}$ & [Martens et al., 2005] & & $\begin{array}{l}\text { [Monge-Rojas et al., 2002; } \\
\text { Videon et al., 2003] }\end{array}$ \\
\hline Compulsão, Insatisfação corporal e TP & & [Peltzer \& Pengpid, 2010] & [Peltzer \& Pengpid, 2012] \\
\hline Influência da imagem corporal & & [Videon et al., 2003] & \\
\hline \multicolumn{4}{|l|}{ Hábito alimentar do adolescente } \\
\hline Refeições irregulares & & [Pedersen et al., 2012H/M] & \\
\hline Realizar as refeições fora de casa/escola & [Lachat et al., 2009] & & \\
\hline \multicolumn{4}{|l|}{ Meio de acesso as informações } \\
\hline Comerciais de rádio & & [Freisling et al., 2010] & \\
\hline Artigos de jornais/ livretos & [Freisling et al., 2010] & & \\
\hline Artigos de Revistas & [Freisling et al., 2010] & & \\
\hline Internet & [Freisling et al., 2010] & & \\
\hline \multicolumn{4}{|l|}{ Influência familiar } \\
\hline $\begin{array}{l}\text { Alimentação saudável dos pais/ } \\
\text { Frequência de refeições }\end{array}$ & $\begin{array}{l}\text { [Fulkerson et al., 2009; Verzeletti } \\
\text { et al., 2010; Videon et al., 2003; } \\
\text { Neumark-Sztainer et al., 2003] }\end{array}$ & & $\begin{array}{l}\text { [Arcan et al., 2007; Burgess- } \\
\text { Champoux et al., 2009] }\end{array}$ \\
\hline Falta de ligação afetiva com os pais & & [Peltzer \& Pengpid, 2010] & [Videon et al., 2003] \\
\hline Apoio familiar para alimentação saudável & & & [Monge-Rojas et al., 2010] \\
\hline Ingestão de frutas pela família & [Arcan et al., 2007] & & [Martens et al., 2005] \\
\hline Ingestão de verduras pela família & [Arcan et al., 2007] & & \\
\hline Morar com os pais & [Riediger et al., 2007] & & [Moraes et al., 2012] \\
\hline Regras alimentares impostas pelos pais & $\begin{array}{l}\text { [Kremers et al., 2003; Verzeletti } \\
\text { et al., 2010] }\end{array}$ & & [Martens et al., 2005] \\
\hline \multicolumn{4}{|l|}{$\begin{array}{l}\text { Disponibilidade e acessibilidade aos } \\
\text { alimentos }\end{array}$} \\
\hline Disponibilidade de alimentos saudáveis & & & $\begin{array}{l}\text { [Arcan et al., 2007; Martens et } \\
\text { al., 2005] }\end{array}$ \\
\hline \multicolumn{4}{|l|}{ Fatores socioeconômicos } \\
\hline Renda familiar & $\begin{array}{l}\text { [Riediger et al., 2007; Giskes et al., } \\
\text { 2002; Assumpção et al., 2012] }\end{array}$ & & $\begin{array}{l}\text { [Fernandes et al., 2011; Freisling } \\
\text { et al., 2010; Fulkerson et al., } \\
\text { 2009; Monge-Rojas et al., 2002; } \\
\text { Moraes et al., 2010] }\end{array}$ \\
\hline Status socioeconômico médio ou alto & [Verzeletti et al., 2010] & & $\begin{array}{l}\text { [Ortiz-Hernández \& Gómez-Tello, } \\
\text { 2008; Kourlaba et al., 2009] }\end{array}$ \\
\hline Ter passado fome & & [Peltzer \& Pengpid, 2010] & [Peltzer \& Pengpid, 2012] \\
\hline
\end{tabular}


Tabela 2 (continuação)

\begin{tabular}{|c|c|c|c|}
\hline \multirow{2}{*}{ Fatores estudados } & \multicolumn{3}{|c|}{ Referências } \\
\hline & Associações positivas & Associações inversas & Sem associação \\
\hline \multicolumn{4}{|l|}{ Fatores demográficos } \\
\hline Sexo & [Shi et al., 2005M] & $\begin{array}{l}\text { [Peltzer \& Pengpid, } 2010 \mathrm{H} ; \\
\text { Moraes et al., 2010H] }\end{array}$ & $\begin{array}{l}\text { [Assumpção et al., 2012; } \\
\text { Fernandes et al., 2011; Freisling } \\
\text { et al., 2010; Fulkerson et al., } \\
\text { 2009; Gerrits et al., 2010; } \\
\text { Kourlaba et al., 2009; Lien et } \\
\text { al., 2002; Martens et al., 2005; } \\
\text { Monge-Rojas et al., 2002; Shi } \\
\text { et al., 2010; Peltzer \& Pengpid, } \\
\text { 2012; Videon et al., 2003; } \\
\text { Verzeletti et al., 2010] }\end{array}$ \\
\hline Idade & [Verzeletti et al., 2010] & [Pedersen et al., 2012] & $\begin{array}{l}\text { [Assumpção et al., 2012; Bigio } \\
\text { et al., 2011; Farias et al., 2009; } \\
\text { Burgess-Champoux et al., 2009; } \\
\text { Fernandes et al., 2011; Freisling } \\
\text { et al., 2010; Fulkerson et al., } \\
\text { 2009; Gerrits et al., 2010; Ortiz- } \\
\text { Hernández \& Gómez-Tello, 2008; } \\
\text { Martens et al., 2005; Monge- } \\
\text { Rojas et al., 2002; Moraes et al., } \\
\text { 2010; Neumark-Sztainer et al., } \\
\text { 1996; Peltzer \& Pengpid, 2010; } \\
\text { Peltzer \& Pengpid, 2012; Videon } \\
\text { et al., 2003] }\end{array}$ \\
\hline Escolaridade dos pais & $\begin{array}{l}\text { [Ball et al., 2009; Riediger } \\
\text { et al., 2007; Videon et al., 2003; } \\
\text { Assumpção et al., 2012] }\end{array}$ & & \\
\hline Etnia Negra & [Videon et al., 2003] & & $\begin{array}{l}\text { [Assumpção et al., 2012; Burgess- } \\
\text { Champoux et al., 2009; Freisling } \\
\text { et al., 2010; Fulkerson et al., } \\
\text { 2009; Riediger et al., 2007] }\end{array}$ \\
\hline Escolaridade do adolescente & & [Peltzer \& Pengpid, 2010] & [Fulkerson et al., 2009] \\
\hline Estudar e trabalhar & & $\begin{array}{l}\text { [Ortiz-Hernández \& Gómez-Tello, } \\
\text { 2008] }\end{array}$ & \\
\hline Idade do chefe da família & $\begin{array}{l}\text { [Ortiz-Hernández \& Gómez-Tello, } \\
\text { 2008] }\end{array}$ & & \\
\hline
\end{tabular}

TP: Transtornos Psicológicos; H/M: Homens e Mulheres analisados separadamente.

Tabela 3. Fatores associados ao consumo de verduras de adolescentes (10 a 19 anos).

\begin{tabular}{|c|c|c|c|}
\hline \multirow{2}{*}{ Fatores estudados } & \multicolumn{3}{|c|}{ Referências } \\
\hline & Associações positivas & Associações inversas & Sem associação \\
\hline \multicolumn{4}{|l|}{ Comportamentos de risco e de saúde } \\
\hline Uso de álcool & & [Roos et al., 2001H/M] & $\begin{array}{l}\text { [Bigio et al., 2011; Peltzer \& } \\
\text { Pengpid, 2012] }\end{array}$ \\
\hline Uso de tabaco & & $\begin{array}{l}\text { [Freisling et al., 2010; Roos et al., } \\
2001 \mathrm{H} / \mathrm{M} \text { ] }\end{array}$ & $\begin{array}{l}\text { [Kourlaba et al., 2009; Lien et al., } \\
\text { 2002; Peltzer \& Pengpid, 2012] }\end{array}$ \\
\hline Comportamento sedentário & & $\begin{array}{l}\text { [Vereecken et al., 2006; Freisling } \\
\text { et al., 2010; Verzeletti et al., } \\
\text { 2010; Roos et al., } 2001 \mathrm{H} / \mathrm{M}]\end{array}$ & [Fernandes et al., 2011] \\
\hline Ser ativo & [Fernandes et al., 2011] & & \\
\hline \multicolumn{4}{|l|}{ Peso corporal e Percepções } \\
\hline Estar abaixo do peso & & $\begin{array}{l}\text { [Moraes et al., 2010; Roos et al., } \\
\text { 2001M] }\end{array}$ & \\
\hline Estar acima do peso & & [Roos et al., 2001H/M] & [Neumark-Sztainer et al., 1996] \\
\hline Desempenho escolar ruim & & [Roos et al., 2001H/M] & \\
\hline Influência da imagem corporal & & $\begin{array}{l}\text { [Videon et al., 2003; Roos et al., } \\
2001 \mathrm{H} / \mathrm{M} \text { ] }\end{array}$ & \\
\hline
\end{tabular}


Tabela 3 (continuação)

\begin{tabular}{|c|c|c|c|}
\hline \multirow{2}{*}{ Fatores estudados } & \multicolumn{3}{|c|}{ Referências } \\
\hline & Associações positivas & Associações inversas & Sem associação \\
\hline \multicolumn{4}{|l|}{ Hábito alimentar do adolescente } \\
\hline Refeições irregulares & & [Pedersen et al., $2012 \mathrm{H}$ & \\
\hline Frequência de refeições diárias & [Arcan et al., 2007] & & \\
\hline Refeições fora de casa/escola & & [Roos et al., 2001H/M] & [Lachat et al., 2009] \\
\hline Comer salgadinhos na escola & & [Roos et al., 2001H] & \\
\hline
\end{tabular}

Meio de acesso às informações

Comerciais de TV

Artigos de jornais/livretos

Internet

Influência familiar

Alimentação saudável dos

pais/Frequência de refeições

Envolvimento do pai que mora fora

Ingestão de frutas pela família

Ingestão de verduras pela família

Morar com os pais

Regras alimentares impostas pelos pais

Morar sozinho

Ter pai, mãe ou ambos fumantes

Influência de amigos e colegas

Ingestão de verduras pelos amigos

Não ter amigos próximos

Fatores socioeconômicos

Renda familiar

[Riediger et al., 2007;

Giskes et al., 2002]

Status socioeconômico médio ou alto

[Verzeletti et al., 2010]

Status socioeconômico baixo

Pais desempregados

Fatores demográficos

Sexo

Burgess-Champoux et al $2009 \mathrm{H} / \mathrm{M}$; Verzeletti et al., 2010]

[Freisling et al., 2010]

[Freisling et al., 2010]

[Arcan et al., 2007; Videon et al.,

2003; Verzeletti et al., 2010;

Roos et al., 2001H/M; Burgess-

Sztainer et al., 2003]

[Stewart \& Menning, 2009]

[Arcan et al., 2007]

[Arcan et al., 2007.

Hanson et al., 2005]

[Riediger et al., 2007]

[Stewart \& Menning, 2009]

[Bruening et al., 2012]

[Freisling et al., 2010]

[Moraes et al., 2012]

[Roos et al., 2001H/M]

[Fulkerson et al., 2009]

[Peltzer \& Pengpid, 2010] 
Tabela 3 (continuação)

\begin{tabular}{|c|c|c|c|}
\hline \multirow{2}{*}{ Fatores estudados } & \multicolumn{3}{|c|}{ Referências } \\
\hline & Associações positivas & Associações inversas & Sem associação \\
\hline \multicolumn{4}{|l|}{ Fatores demográficos } \\
\hline Escolaridade dos pais & $\begin{array}{l}\text { [Stewart \& Menning, 2009; } \\
\text { Riediger et al., 2007; Videon et al., } \\
\text { 2003; Assumpção et al., 2012] }\end{array}$ & & \\
\hline Etnia Negra & [Stewart \& Menning, 2009] & [Videon et al., 2003] & $\begin{array}{l}\text { [Assumpção et al., 2012; Burgess- } \\
\text { Champoux et al., 2009; Freisling } \\
\text { et al., 2010; Fulkerson et al., } \\
\text { 2009; Riediger et al., 2007] }\end{array}$ \\
\hline Escolaridade do adolescente & [Peltzer \& Pengpid, 2010] & & [Fulkerson et al., 2009] \\
\hline
\end{tabular}

H/M: Homens e Mulheres analisados separadamente.

Tabela 4. Fatores associados ao consumo de frutas e verduras de adolescentes (10 a 19 anos).

\begin{tabular}{|c|c|c|c|}
\hline \multirow{2}{*}{ Fatores estudados } & \multicolumn{3}{|c|}{ Referências } \\
\hline & Associações positivas & Associações inversas & Sem associação \\
\hline \multicolumn{4}{|l|}{ Comportamentos de risco e de saúde } \\
\hline Uso de álcool & & [Neumark-Sztainer et al., 1996] & $\begin{array}{l}\text { [Bigio et al., 2011; Peltzer \& } \\
\text { Pengpid, 2012] }\end{array}$ \\
\hline Uso de drogas & & [Neumark-Sztainer et al., 1996] & [Peltzer \& Pengpid, 2012] \\
\hline Uso de tabaco & & $\begin{array}{l}\text { [Bigio et al., 2011; Neumark- } \\
\text { Sztainer et al., 1996] }\end{array}$ & $\begin{array}{l}\text { [Kourlaba et al., 2009; Lien et al., } \\
\text { 2002; Peltzer \& Pengpid, 2012] }\end{array}$ \\
\hline Comportamento sedentário & & $\begin{array}{l}\text { [Shi et al., 2010; Peltzer \& Pengpid, } \\
\text { 2012; Kourlaba et al., 2009] }\end{array}$ & [Fernandes et al., 2011] \\
\hline Ser ativo & $\begin{array}{l}\text { [Fernandes et al., 2011; Lien et al., } \\
\text { 2002; Moraes et al., 2012] }\end{array}$ & & \\
\hline Praticar esportes fora da escola & [Kourlaba et al., 2009] & & \\
\hline
\end{tabular}

Peso corporal e Percepções

\begin{tabular}{|c|c|}
\hline Estar acima do peso & [Peltzer \& Pengpid, 2012] \\
\hline $\begin{array}{l}\text { Habilidade para escolher alimentos } \\
\text { saudáveis }\end{array}$ & [Young et al., 2004] \\
\hline Autocontrole/ Boa saúde e dieta & $\begin{array}{l}\text { [Lien et al., 2002; Gerrits et al., } \\
\text { 2010] }\end{array}$ \\
\hline \multicolumn{2}{|l|}{ Compulsão, Insatisfação corporal e TP } \\
\hline \multicolumn{2}{|l|}{ Desempenho escolar ruim } \\
\hline Influência da imagem corporal & [Monge-Rojas et al., 2002] \\
\hline \multicolumn{2}{|l|}{ Hábito alimentar do adolescente } \\
\hline Frequência de refeições diárias & $\begin{array}{l}\text { [Arcan et al., 2007; Lien et al., } \\
\text { 2002] }\end{array}$ \\
\hline \multicolumn{2}{|l|}{ Dieta mais de cinco vezes ao ano } \\
\hline \multicolumn{2}{|l|}{ Influência familiar } \\
\hline $\begin{array}{l}\text { Alimentação saudável dos pais/ } \\
\text { Frequência de refeições }\end{array}$ & $\begin{array}{l}\text { [Neumark-Sztainer et al., 2003; } \\
\text { Hanson et al., 2005H/M; Cutler et } \\
\text { al., 2011; Gerrits et al., 2010; Lien } \\
\text { et al., 2002; Monge-Rojas et al., } \\
\text { 2002; Moraes et al., 2012; Young } \\
\text { et al., 2004] }\end{array}$ \\
\hline Ligação afetiva com os pais & $\begin{array}{l}\text { [Lien et al., 2002; Peltzer \& } \\
\text { Pengpid, 2010; Neumark-Sztainer } \\
\text { et al., 1996] }\end{array}$ \\
\hline Apoio familiar para boa alimentação & $\begin{array}{l}\text { [Cutler et al., 2011; Young et al., } \\
\text { 2004; Peltzer \& Pengpid, 2012] }\end{array}$ \\
\hline Ingestão de frutas pela família & $\begin{array}{l}\text { [Arcan et al., 2007; Hanson et al., } \\
2005 \mathrm{M}]\end{array}$ \\
\hline
\end{tabular}

[Monge-Rojas et al., 2002; Fernandes et al., 2011]

[Neumark-Sztainer et al., 1996] [Peltzer \& Pengpid, 2012]

[Neumark-Sztainer et al., 1996]

[Neumark-Sztainer et al., 1996] 
Tabela 4 (continuação)

\begin{tabular}{|c|c|c|c|}
\hline \multirow{2}{*}{ Fatores estudados } & \multicolumn{3}{|c|}{ Referências } \\
\hline & Associações positivas & Associações inversas & Sem associação \\
\hline \multicolumn{4}{|l|}{ Influência familiar } \\
\hline Ingestão de verduras pela família & $\begin{array}{l}\text { [Arcan et al., 2007; Hanson et al., } \\
\text { 2005M] }\end{array}$ & & \\
\hline Morar com os pais & [Shi et al., 2010] & & [Moraes et al., 2012] \\
\hline Sanções verbais e restrição alimentar & & [Monge-Rojas et al., 2010H] & \\
\hline Refeições com a família em casa & [Moraes et al., 2012M] & & \\
\hline Café da manhã com a família em casa & [Moraes et al., 2012H] & & \\
\hline \multicolumn{4}{|l|}{ Influência de amigos e colegas } \\
\hline Apoio/relações positivas com colegas & $\begin{array}{l}\text { [Monge-Rojas et al., 2002; Lien et } \\
\text { al., 2002; Cutler et al., 2011] }\end{array}$ & & \\
\hline \multicolumn{4}{|l|}{ Disponibilidade e acesso aos alimentos } \\
\hline Disponibilidade de alimentos saudáveis & $\begin{array}{l}\text { [Cutler et al., 2011; Hanson et al., } \\
\text { 2005M; Young et al., 2004] }\end{array}$ & & \\
\hline Alimentos não saudáveis & & [Cutler et al., 2011] & \\
\hline \multicolumn{4}{|l|}{ Fatores socioeconômicos } \\
\hline Renda familiar & $\begin{array}{l}\text { [Beydoun et al., 2011; Bigio et al., } \\
\text { 2011; Cutler et al., 2011; Lien et } \\
\text { al., 2002; Shi et al., 2005] }\end{array}$ & [Farias et al., 2009] & \\
\hline Status socioeconômico ruim & & [Neumark-Sztainer et al., 1996] & [Shi et al., 2010] \\
\hline Preço dos alimentos não saudáveis & [Beydoun et al., 2011] & & \\
\hline \multicolumn{4}{|l|}{ Fatores demográficos } \\
\hline Sexo & $\begin{array}{l}\text { [Neumark-Sztainer et al., 1996; } \\
\text { Lachat et al., 2009H; Shi et al., } \\
\text { 2005] }\end{array}$ & [Farias et al., 2009H] & \\
\hline Idade & [Shi et al., 2010] & [Kourlaba et al., 2009] & $\begin{array}{l}\text { [Assumpção et al., 2012; Bigio } \\
\text { et al., 2011; Farias et al., 2009; } \\
\text { Burgess-Champoux et al., 2009; } \\
\text { Fernandes et al., 2011; Freisling } \\
\text { et al., 2010; Fulkerson et al., } \\
\text { 2009; Gerrits et al., 2010; Ortiz- } \\
\text { Hernández \& Gómez-Tello, 2008; } \\
\text { Monge-Rojas et al., 2002; Moraes } \\
\text { et al., 2010; Neumark-Sztainer } \\
\text { et al., 1996; Peltzer \& Pengpid, } \\
\text { 2010; Peltzer \& Pengpid, 2012; } \\
\text { Videon et al., 2003] }\end{array}$ \\
\hline Escolaridade dos pais & $\begin{array}{l}\text { [Bigio et al., 2011; Monge-Rojas et } \\
\text { al., 2002; Shi et al., 2010; Shi et } \\
\text { al., 2005] }\end{array}$ & $\begin{array}{l}\text { [Ortiz-Hernández \& Gómez-Tello, } \\
\text { 2008] }\end{array}$ & \\
\hline Raça/ Etnia & $\begin{array}{l}\text { [Cutler et al., 2011; Shi et al., } \\
\text { 2010] }\end{array}$ & & $\begin{array}{l}\text { [Assumpção et al., 2012; Burgess- } \\
\text { Champoux et al., 2009; Freisling } \\
\text { et al., 2010; Fulkerson et al., } \\
\text { 2009; Riediger et al., 2007] }\end{array}$ \\
\hline Morar em área rural & [Monge-Rojas et al., 2002] & & \\
\hline Morar em área urbana & & $\begin{array}{l}\text { [Farias et al., 2009; Shi et al., } \\
2005 \mathrm{H} \text { ] }\end{array}$ & \\
\hline Trabalhar & & $\begin{array}{l}\text { [Ortiz-Hernández \& Gómez-Tello, } \\
\text { 2008; Farias et al., 2009] }\end{array}$ & \\
\hline Não estudar e nem trabalhar & & $\begin{array}{l}\text { [Ortiz-Hernández \& Gómez-Tello, } \\
\text { 2008] }\end{array}$ & \\
\hline Numero de irmãos & [Kourlaba et al., 2009] & & \\
\hline Menos de três membros na família & [Shi et al., 2005] & & \\
\hline
\end{tabular}

TP: Transtornos Psicológicos; H/M: Homens e Mulheres analisados separadamente. 


\section{Comportamentos de risco e de saúde dos adolescentes}

Destacam-se predominantemente: comportamento sedentário (9 estudos), uso de tabaco (8 estudos) e álcool (4 estudos). O uso de drogas e a inatividade física também foram alvos de investigação. Adolescentes que passaram mais tempo em atividades sedentárias, que relataram uso de tabaco e álcool, apresentaram significativamente menores consumos de frutas e verduras. Entretanto a prática de atividade física constituiu um fator de influência positiva sobre a dieta adequada.

\section{Peso corporal e percepção dos adolescentes}

Este tema envolveu a percepção dos adolescentes quanto à alimentação, atitudes dos pais e professores, fatores psicológicos, além de percepção corporal e do próprio peso. A habilidade dos adolescentes em escolher alimentos saudáveis esteve entre os fatores mais investigados ${ }^{22,25} \mathrm{e}$ representou um fator associado ao consumo de frutas e verduras. Adolescentes com autocontrole e boa avaliação da própria saúde e dieta ${ }^{31,53}$ consumiam mais frutas e verduras. Com exceção de um estudo ${ }^{43}$, estar abaixo ou acima do peso corporal foi prejudicial, assim como a insatisfação corporal, compulsão alimentar, transtornos psicológicos e desempenho escolar ruim.

\section{Hábito alimentar dos adolescentes}

A frequência alimentar, estabelecida pelo número de refeições, esteve entre os fatores de maior influência positiva sobre a ingestão de frutas e verduras. Adolescentes que realizavam as refeições fora de casa tiveram melhor consumo de frutas $^{49}$, mas não de verduras ${ }^{41}$. Contrapondo-se a isso, refeições irregulares ${ }^{46}$, comer salgadinhos durante o dia na escola ${ }^{41}$ e fazer dieta mais de cinco vezes ao $\mathrm{ano}^{45}$, são fatores que prejudicaram o consumo destes alimentos. Observou-se que a preocupação com estes fatores não é recente, entretanto poucos trabalhos têm investigado estes aspectos.

\section{Meio de acesso às informações sobre alimentação}

Seis meios de acesso a informações sobre alimentação foram estudados ${ }^{36}$. Adolescentes que tiveram acesso a informações veiculadas em jornais/livretos, revistas e internet apresentaram melhor consumo de frutas e verduras, já o acesso às informações obtidas em comerciais de rádio ou TV contribuiu para menor ingestão destes alimentos.

\section{Influência familiar}

Os fatores mais prevalentes foram: ingestão alimentar dos pais/ frequência de refeições da família $(n=14)$; ligação afetiva com os pais $(n=4)$; apoio familiar para alimentação saudável $(n=4)$. O consumo de frutas e verduras pelos pais ${ }^{26,30} \mathrm{e}$ o apoio à alimentação saudável dos filhos, foram potenciais fatores associados à ingestão de frutas e verduras. O uso de sanções verbais pelos pais para encorajar a alimentação saudável diminuiu o consumo de frutas ${ }^{32}$, mas ter pais que impuseram regras de restrição e obrigação alimentar contribuiu para o consumo de frutas e verduras ${ }^{24,37}$. Esta associação positiva também foi encontrada quando o adolescente morava com pai e mãe $\mathrm{e}^{23,38}$.

\section{Influência de amigos, colegas e apoio social}

A ingestão de verduras pelos amigos influenciou positivamente o consumo destes alimentos em adolescentes ${ }^{27}$ já a ausência de amigos próximos prejudicou o consumo ${ }^{42}$. A influência do ambiente social, relações positivas com os colegas e o apoio dos colegas para uma alimentação saudável foram importantes contribuintes no consumo de frutas e verduras de adolescentes $29,31,39$.

\section{Disponibilidade e acessibilidade aos alimentos}

Este tema incluiu o relato de disponibilidade de frutas e verduras, de alimentos saudáveis e de alimentos não saudáveis no domicilio, além de acessibilidade aos alimentos. Os fatores foram investigados por cinco trabalhos e destes, três 25, 29,30 identificaram melhor consumo de frutas e verduras quando os adolescentes reportaram ter alimentos saudáveis disponíveis em casa. Quando relataram a disponibilidade de alimentos não saudáveis, o consumo de frutas e verduras foi significativamente menor ${ }^{29}$.

\section{Fatores socioeconômicos}

A renda familiar foi o fator mais estudado $(n=15)$, seguido do status socioeconômico $(n=5)$. Associações positivas foram encontradas entre renda e consumo de frutas e verduras pela maioria das investigações, sendo assim, adolescentes com maior renda familiar apresentaram melhor ingestão desses alimentos. Os resultados para status socioeconômico são divergentes. O preço de alimentos considerados fast food ${ }^{20}$ e ter um dos pais ou ambos desempregados ${ }^{41}$ estiveram inversamente associados ao consumo de frutas e verduras e de verduras, respectivamente.

\section{Fatores demográficos}

Sexo e idade dos adolescentes, seguido pela escolaridade dos pais e etnia (adolescentes) representaram a maior parcela de investigações. A ingestão inadequada de frutas e verduras esteve consistentemente associada ao sexo masculino, porém quanto maior a escolaridade dos pais, melhor foi a ingestão desses alimentos. Adolescentes que trabalhavam ${ }^{21,48}$ 
e estudavam ${ }^{21}$ ou que não trabalhavam nem estudavam ${ }^{21}$ apresentaram baixo consumo de frutas e verduras, assim como aqueles que residiam em área urbana ${ }^{48,50}$, comparados aos que residiam em área rural ${ }^{39}$.

\section{DISCUSSÃO}

A maior parte das investigações está centrada em comportamentos de risco à saúde, fatores do aspecto social como a influência familiar, além de fatores socioeconômicos e demográficos. Adolescentes de maior renda familiar consomem mais frutas e verduras ${ }^{14,20,23,29,31,40,50,52}$. Por outro lado, a situação de insegurança alimentar afeta negativamente o consumo de frutas e verduras ${ }^{42}$. Frutas e verduras tendem a ter maior custo comparado aos alimentos considerados não saudáveis e, talvez não representem a prioridade de famílias em situações de insegurança alimentar. Além disso, a moradia em área urbana pode impossibilitar a oferta de uma variedade de alimentos saudáveis com preços acessíveis, visto que a moradia em área rural ${ }^{39}$ proporciona maior variedade de alimentos saudáveis comparado a moradia em região urbana ${ }^{48,50}$.

A identificação das condições socioeconômicas e demográficas auxilia no direcionamento de intervenções nutricionais para a população alvo, como a população de baixa renda e em situações de insegurança alimentar. Neste sentido, deve-se considerar não apenas o aumento do poder aquisitivo, mas o conhecimento na escolha dos alimentos, tendo em vista a influência negativa da baixa escolaridade paterna sobre o consumo de frutas e verduras dos adolescentes ${ }^{25,29,30}$. O alto nível educacional dos pais se destaca como fator de influência positiva para alimentação saudável dos adolescentes ${ }^{14,23,38,39,44,47,50,52,57}$. Pais com maior nível educacional possuem maior conhecimento de doenças crônicas e priorizam o estilo de vida saudável, consequentemente as escolhas alimentares dos pais refletem nos hábitos dos filhos $24-26,28-31,34,39,41,47,51,53,56$. Além disso, o nível educacional dos pais influencia o nível de educação nutricional dos adolescentes quanto a habilidade para escolher alimentos saudáveis ${ }^{22,25}$, o que reflete no consumo de frutas e verduras.

As regras de restrição e obrigação alimentar impostas pela família $^{24,37}$, e a frequência de realização das refeições ${ }^{26,31}$, estão associadas ao maior consumo de frutas e verduras, quando analisados separadamente. Este resultado indica a importância de avaliar o consumo desses alimentos em diferentes condições, pois frutas geralmente são consumidas como sobremesa ou lanche entre as refeições, e verduras são consumidas como parte de uma grande refeição. Nesse sentido, as regras de restrição a lanches entre as refeições podem influenciar o consumo de frutas enquanto as regras impostas durante almoço e jantar influenciam o consumo de verduras.

O ambiente social do adolescente representa um fator de influência pouco investigado. Contudo, igualmente importante, considerando que um bom relacionamento pessoal e o apoio dos colegas representam um incentivo para alimentação saudáve $\left.\right|^{29,31,39}$. No ambiente escolar os adolescentes sofrem a influência de amigos e colegas, com isso, ações de educação em saúde podem orientar quanto ao risco do uso de tabaco, bebida alcoólica, comportamentos sedentários e inatividade física. Considerando a relação entre habilidade para escolha de alimentos saudáveis e maior consumo de frutas e verduras, as escolas precisam abranger temas relacionados à alimentação saudável de maneira a contribuir na adoção de escolhas conscientes. A inserção destes temas nos conteúdos curriculares seria uma forma de garantir estas abordagens.

O suporte familiar também é importante nos hábitos alimentares dos filhos. Estudos que analisaram este fator de influência concluíram que a ligação afetiva dos adolescentes com os pais pode aumentar a ingestão de frutas e verduras $\mathrm{s}^{42,57}$. As relações familiares refletem a adoção de um estilo de vida saudável e prevenção de comportamentos de risco a saúde como beber, fumar, comportamento sedentário ou fisicamente inativo, que são reconhecidamente um impacto negativo sobre a ingestão de frutas e verduras ${ }^{14,24,35,36,41-43,55}$. É importante que programas de promoção à alimentação saudável sejam estendidos à família, uma vez que há evidências da participação dos pais e da família como um fator chave no comportamento dos adolescentes. A família deve ser incentivada a ser modelo positivo, aumentando a frequência das refeições familiares e o nível de suporte afetivo para o enfrentamento de situações adversas no período de transição da adolescência para a idade adulta.

Observa-se a necessidade de articulação entre instituições de ensino e setores da saúde para incorporação de ações individuais, no âmbito familiar e comunitário. $\mathrm{O}$ acesso aos alimentos saudáveis poderia ser garantido através da produção de hortas na comunidade e/ou escola. Setores governamentais, ao desenvolver ações organizadas, podem intervir sobre fatores dificilmente atingíveis como a influência midiática, para atuar no controle da publicidade de alimentos pobres em nutrientes, e incentivo a campanhas que estimulem a alimentação saudável.

Os estudos apresentados foram conduzidos majoritariamente nos Estados Unidos, por isso, considera-se que diferenças econômicas, sociais e culturais possam influenciar a identificação de aspectos relacionados à alimentação. Nesse sentido, os resultados apresentados sugerem potenciais campos de pesquisa para a investigação 
dos hábitos alimentares de adolescentes brasileiros, considerando que os achados denotam complexidade na compreensão do comportamento alimentar. A identificação de comportamentos modificáveis durante a adolescência pode garantir a adoção de hábitos alimentares saudáveis na vida adulta.

De forma geral, os fatores socioeconômicos, demográficos, relacionados ao estilo de vida, e a influência da família e dos amigos representam potenciais fatores associados à ingestão de frutas e verduras na adolescência. Considerando que o comportamento alimentar dos adolescentes é formado em meio a múltiplos determinantes, estes resultados destacam a importância de ampliar as abordagens de intervenção para alcançar mais de uma dimensão de influência. Os fatores aqui discutidos deveriam ser integrados a programas de promoção/educação à saúde para aumentar o consumo diário de frutas e verduras entre adolescentes.

\section{REFERÊNCIAS}

1. Patton GC, Coffey C, Cappa C, Currie D, Riley L, Gore F, Degenhardt L, Richardson D, Astone N, Sangowawa AO, Mokdad A, Ferguson J. Health of the world's adolescents: a synthesis of internationally comparable data. Lancet. 2012;379(9826):1665-75. https://doi. org/10.1016/S0140-6736(12)60203-7

2. Ledoux TA, Hingle MD, Baranouwski T. Relationship of fruit and vegetable intake with adiposity: a systematic review. Obes Rev. 2011;12(5):e143-50. https://doi.org/10.1111/j.1467789X.2010.00786.x

3. Lock K, Pomerleau J, Causer L, Altmann DR, Mckee M. The global burden of disease attributable to low consumption of fruit and vegetables: implications for the global strategy on diet. Bull World Health Organ. 2005;83(2):100-8.

4. Zhang X, Shu XO, Xiang YB, Yang G, Li H, Gao J, Cai H, Gao YT, Zheng W. Cruciferous vegetable consumption is associated with a reduced risk of total and cardiovascular disease mortality. Am J Clin Nutr. 2011;94(1):240-6. https://doi.org/10.3945/ajcn.110.009340

5. Crowe FL, Roddam AW, Key TJ, Appleby PN, Overvad K, Jakobsen MU, Tjønneland A, Hansen L, Boeing $H$, Weikert C, Linseisen J, Kaaks R, Trichopoulou A, Misirli G, Lagiou P, Sacerdote C, Pala V, Palli D, Tumino R, Panico S, Bueno-de-Mesquita HB, Boer J, van Gils $\mathrm{CH}$, Beulens JW, Barricarte A, Rodríguez L, Larra-aga N, Sánchez MJ, Tormo MJ, Buckland G, Lund E, Hedblad B, Melander O, Jansson JH, Wennberg P, Wareham NJ, Slimani N, Romieu I, Jenab M, Danesh J, Gallo V, Norat T, Riboli E; European Prospective Investigation into Cancer and Nutrition (EPIC)-Heart Study Collaborators. Fruit and vegetable intake and mortality from ischaemic heart disease: results from the European Prospective Investigation into Cancer and Nutrition (EPIC)-Heart study. Eur Heart J. 2011;32(10): 1235-43. https://doi.org/10.1093/eurheartj/ehq465

6. Epplein M, Shu XO, Xiang YB, Chow WH, Yang G, Li HL, Cai H, Gao YT, Zheng W. Fruit and vegetable consumption and risk of distal gastric cancer in the Shanghai Women's and Men's Health studies. Am J Epidemiol. 2010;172(4):397-406. https://doi.org/10.1093/aje/ kwq144
7. Boffetta P, Couto E, Wichmann J, Ferrari P, Trichopoulos D, Buenode-Mesquita HB, van Duijnhoven FJ, Büchner FL, Key T, Boeing H, Nöthlings U, Linseisen J, Gonzalez CA, Overvad K, Nielsen MR, Tjønneland A, Olsen A, Clavel-Chapelon F, Boutron-Ruault MC, Morois S, Lagiou P, Naska A, Benetou V, Kaaks R, Rohrmann S, Panico $\mathrm{S}$, Sieri S, Vineis P, Palli D, van Gils $\mathrm{CH}$, Peeters PH, Lund E, Brustad $M$, Engeset D, Huerta JM, Rodríguez L, Sánchez MJ, Dorronsoro M, Barricarte A, Hallmans G, Johansson I, Manjer J, Sonestedt E, Allen NE, Bingham S, Khaw KT, Slimani N, Jenab M, Mouw T, Norat T, Riboli E, Trichopoulou A. Fruit and vegetable intake and overall cancer risk in the European Prospective Investigation Into Cancer and Nutrition (EPIC). J Natl Cancer Inst. 2010;102(8):529-37. https://doi.org/10.1093/jnci/djq072

8. Wakai K, Matsuo K, Nagata C, Mizoue T, Tanaka K, Tsuji I, Sasazuki S, Shimazu T, Sawada N, Inoue M, Tsugane S; Research Group for the Development and Evaluation of Cancer Prevention Strategies in Japan. Lung cancer risk and consumption of vegetables and fruit: an evaluation based on a systematic review of epidemiological evidence from Japan. Jpn J Clin Oncol. 2011;41(5):693-708. https:// doi.org/10.1093/jjco/hyr027

9. McNaughton SA, Ball K, Mishra GD, Crawford DA. Dietary patterns of adolescents and risk of obesity and hypertension. J Nutr. 2008;138(2):364-70. https://doi.org/10.1093/jn/138.2.364

10. Cutler GJ, Flood A, Hannan PJ, Slavin JL, Neumark-Sztainer D. Association between major patterns of dietary intake and weight status in adolescents. Br J Nutr. 2012;108(2):349-56. https://doi. org/10.1017/S0007114511005435

11. Liu JH, Chu YH, Frongillo EA, Probst JC. Generation and acculturation status are associated with dietary intake and body weight in Mexican American adolescents. J Nutr. 2012;142(2):298-305. https://doi. org/10.3945/jn.111.145516

12. Lien N, Lytle LA, Klepp KI. Stability in consumption of fruit, vegetables and sugary foods in a cohort from age 14 to age 21. Prev Med. 2001;33(3):217-26. https://doi.org/10.1006/pmed.2001.0874

13. Kimmons J, Gillespie C, Seymour J, Serdula M, Blanck HM. Fruit and vegetable intake among adolescents and adults in the United States: percentage meeting individualized recommendations. Medscape J Med. 2009;11(1):26.

14. Bigio RS, Verly Junior E, Castro MA, César CL, Fisberg RM, Marchioni DM. Determinants of fruit and vegetable intake in adolescents using quantile regression. Rev Saude Publ. 2011;45(3):448-56. https:// doi.org/10.1590/S0034-89102011000300002

15. World Health Organization. World Health Assembly 57.17: Global strategy on diet and physical activity. Geneva: WHO; 2004.

16. Centers for Disease Control and Prevention. Strategies to prevent obesity and other chronic diseases: the CDC guide to strategies to increase the consumption of fruits and vegetables. Atlanta: U.S. Department of Health and Human Services; 2011.

17. van der Horst K, Oenema A, Ferreira I, Wendel-Vos W, Giskes K, van Lenthe F, Brug J. A systematic review of environmental correlates of obesity-related dietary behaviors in youth. Health Educ Res. 2007;22(2):203-26. https://doi.org/10.1093/her/cyl069

18. Pearson N, Biddle SJ, Gorely T. Family correlates of fruit and vegetable consumption in children and adolescents: a systematic review. Public Health Nutr. 2009;12(2):267-83. https://doi.org/10.1017/ S1368980008002589 
19. Krølner R, Rasmussen M, Brug J, Klepp KI, Wind M, Due P. Determinants of fruit and vegetable consumption among children and adolescents: a review of the literature. Part II: qualitative studies. Int J Behav Nutr Phys Act. 2011;8:112. https://doi. org/10.1186/1479-5868-8-112

20. Beydoun MA, Powell LM, Chen X, Wang Y. Food prices are associated with dietary quality, fast food consumption, and body mass index among U.S. children and adolescents. J Nutr. 2011;141(2):304-11. https://doi.org/10.3945/jn.110.132613

21. Ortiz-Hernández L, Gómez-Tello BL. Food consumption in Mexican adolescents. Rev Panam Salud Publica. 2008;24(2):127-35. https:// doi.org/10.1590/S1020-49892008000800007

22. Martens MK, van Assema P, Brug J. Why do adolescents eat what they eat? Personal and social environmental predictors of fruit, snack and breakfast consumption among 12-14-year-old Dutch students. Public Health Nutr. 2005;8(8):1258-65. https://doi.org/10.1079/ PHN2005828

23. Riediger ND, Shooshtari S, Moghadasian MH. The influence of sociodemographic factors on patterns of fruit and vegetable consumption in Canadian adolescents. J Am Diet Assoc. 2007;107(9):1511-8. https://doi.org/10.1016/j.jada.2007.06.015

24. Verzeletti C, Maes L, Santinello M, Baldassari D, Vereecken CA. Food-related family lifestyle associated with fruit and vegetable consumption among young adolescents in Belgium Flanders and the Veneto Region of Italy. Appetite. 2010;54(2):394-7. https://doi. org/10.1016/j.appet.2009.12.010

25. Young EM, Fors SW, Hayes DM. Associations between perceived parent behaviors and middle school student fruit and vegetable consumption. J Nutr Educ Behav. 2004;36(1):2-8. https://doi. org/10.1016/S1499-4046(06)60122-X

26. Arcan C, Neumark-Sztainer D, Hannan P, van der Berg P, Story M, Larson N. Parental eating behaviours, home food environment and adolescent intakes of fruits, vegetables and dairy foods: longitudinal findings from Project EAT. Public Health Nutr. 2007;10(11):125765. https://doi.org/10.1017/S1368980007687151

27. Bruening M, Eisenberg M, MacLehose R, Nanney MS, Story M, Neumark-Sztainer D. Relationship between adolescents' and their friends' eating behaviors: breakfast, fruit, vegetable, whole-grain, and dairy intake. J Acad Nutr Diet. 2012;112(10):1608-13. https:// doi.org/10.1016/j.jand.2012.07.008

28. Burgess-Champoux TL, Larson N, Neumark-Sztainer D, Hannan PJ, Story M. Are family meal patterns associated with overall diet quality during the transition from early to middle adolescence? J Nutr Educ Behav. 2009;41(2):79-86. https://doi.org/10.1016/j. jneb.2008.03.113

29. Cutler GJ, Flood A, Hannan PJ, Neumark-Sztainer D. Multiple sociodemographic and socioenvironmental characteristics are correlated with major patterns of dietary intake in adolescents. J Am Diet Assoc. 2011;111(2):230-40. https://doi.org/10.1016/j. jada.2010.10.052

30. Hanson NI, Neumark-Sztainer D, Eisenberg ME, Story M, Wall M. Associations between parental report of the home food environment and adolescent intakes of fruits, vegetables and dairy foods. Public Health Nutr. 2005;8(1):77-85. https://doi.org/10.1079/ PHN2005661
31. Lien N, Jacobs DR Jr, Klepp KI. Exploring predictors of eating behaviour among adolescents by gender and socio-economic status. Public Health Nutr. 2002;5(5):671-81. https://doi.org/10.1079/ PHN2002334

32. Monge-Rojas R, Smith-Castro V, Colon-Ramos U, Garita-Arce C, Sánchez-Lopez M, Chinnock A. Parental feeding styles and adolescents' healthy eating habits. Structure and correlates of a Costa Rican questionnaire. Appetite. 2010;55(2):253-62. https:// doi.org/10.1016/j.appet.2010.06.010

33. de Moraes AC, Fernandes RA, Christofaro DG, Oliveira AR, Nakashima AT, Reichert FF, Falcão MC. Nutrition-related habits and associated factors of Brazilian adolescents. Int J Public Health. 2010;55(6):661-7. https://doi.org/10.1007/s00038-010-0177-9

34. de Moraes AC, Adami F, Falcão MC. Understanding the correlates of adolescents' dietary intake patterns. A multivariate analysis. Appetite. 2012;58(3):1057-62. https://doi.org/10.1016/j.appet.2012.01.024

35. Vereecken CA, Todd J, Roberts C, Mulvihill C, Maes L. Television viewing behaviour and associations with food habits in different countries. Public Health Nutr. 2006;9(2):244-50. https://doi. org/10.1079/PHN2005847

36. Freisling H, Haas K, Elmadfa I. Mass media nutrition information sources and associations with fruit and vegetable consumption among adolescents. Public Health Nutr. 2010;13(2):269-75. https:// doi.org/10.1017/S1368980009991297

37. Kremers SP, Brug J, de Vries H, Engels RC. Parenting style and adolescent fruit consumption. Appetite. 2003;41(1):43-50. https:// doi.org/10.1016/S0195-6663(03)00038-2

38. Shi L, Mao Y. Excessive recreational computer use and food consumption behaviour among adolescents. Ital J Pediatr. 2010;36:52. https://doi.org/10.1186/1824-7288-36-52

39. Monge-Rojas R, Nu-es HP, Garita C, Chen-Mok M. Psychosocial aspects of Costa Rican adolescents' eating and physical activity patterns. J Adoles Health. 2002;31(2):212-9. https://doi. org/10.1016/S1054-139X(02)00376-2

40. Giskes K, Turrell G, Patterson C, Newman B. Socio-economic differences in fruit and vegetable consumption among Australian adolescents and adults. Public Health Nutr. 2002;5(5):663-9. https://doi.org/10.1079/PHN2002339

41. Roos EB, Hirvonen T, Mikkilä V, Karvonem S, Rimpelã M. Household educational level as a determinant of consumption of raw vegetables among male and female adolescents. Prev Med. 2001;33(4):28291. https://doi.org/10.1006/pmed.2001.0882

42. Peltzer K, Pengpid S. Fruits and vegetables consumption and associated factors among in-school adolescents in seven African countries. Int J Public Health. 2010;55(6):669-78. https://doi. org/10.1007/s00038-010-0194-8

43. Peltzer K, Pengpid S. Fruits and vegetables consumption and associated factors among in-school adolescents in five Southeast Asian countries. Int J Environ Res Public Health. 2012;9(10): 3575-87. https://doi.org/10.3390/ijerph9103575

44. Ball K, MacFarlane A, Crawford D, Savige G, Andrianopoulos N, Worsley A. Can social cognitive theory constructs explain socioeconomic variations in adolescent eating behaviours? A mediation analysis. Health Educ Res. 2009;24(3):496-506. https://doi. org/10.1093/her/cyn048 
45. Neumark-Sztainer D, Story M, Resnick MD, Blum RWM. Correlates of inadequate fruit and vegetable consumption among adolescents. Prev Med. 1996;25(5):497-505. https://doi.org/10.1006/pmed. 1996.0082

46. Pedersen TP, Meilstrup C, Holstein BE, Rasmussen M. Fruit and vegetable intake is associated with frequency of breakfast, lunch and evening meal: cross-sectional study of 11-, 13-, and 15-year-olds. Int J Behav Nutr Phys Act. 2012;9:9. https://doi.org/10.1186/14795868-9-9

47. Videon TM, Manning CK. Influences on adolescent eating patterns: the importance of family meals. J Adoles Health. 2003;32(5):36573. https://doi.org/10.1016/S1054-139X(02)00711-5

48. Farias Júnior JC, Nahas MV, Barros MVG, Loch MR, Oliveira ESA, De Bem MFL, Lopes AS. Comportamentos de risco à saúde em adolescentes no Sul do Brasil: prevalência e fatores associados. Rev Panam Salud Publica. 2009;25(4):344-52. https://doi.org/10.1590/ S1020-49892009000400009

49. Lachat C, Khanh le NB, Khan NC, Dung NQ, Nguyen do VA, Roberfroid D, Kolsteren P. Eating out of home in Vietnamese adolescents: socioeconomic factors and dietary associations. Am J Clin Nutr. 2009;90(6):1648-55. https://doi.org/10.3945/ ajcn.2009.28371

50. Shi Z, Lien N, Kumar BN, Holmboe-Ottesen G. Socio-demographic differences in food habits and preferences of school adolescents in Jiangsu Province, China. Eur J Clin Nutr. 2005;59(12):1439-48. https://doi.org/10.1038/sj.ejcn.1602259

51. Fulkerson JA, Kubik MY, Story M, Lytle L, Arcan C. Are there nutritional and other benefits associated with family meals among at-risk youth? J Adoles Health. 2009;45(4):389-95. https://doi. org/10.1016/j.jadohealth.2009.02.011

52. Assumpção D, Barros MBA, Fisberg RM, Carandina L, Goldbaum M, Cesar CLG. Diet quality among adolescents: a population-based study in Campinas, Brazil. Rev Bras Epidemiol. 2012;15(3):605-16. https://doi.org/10.1590/S1415-790X2012000300014

53. Gerrits JH, O'Hara RE, Piko BF, Gibbons FX, de Ridder DT, Keresztes N, Kamble SV, de Wit JB. Self-control, diet concerns and eater prototypes influence fatty foods consumption of adolescents in three countries. Health Educ Res. 2010;25(6):1031-41. https://doi. org/10.1093/her/cyq055

54. Fernandes RA, Christofaro DG, Casonatto J, Kawaguti SS, Ronque ER, Cardoso JR, Freitas Júnior IF, Oliveira AR. Cross-sectional association between healthy and unhealthy food habits and leisure physical activity in adolescents. J Pediatr (Rio J). 2011;87(3):252-6. https://doi.org/10.2223/JPED.2093

55. Kourlaba G, Panagiotakos DB, Mihas K, Alevizos A, Marayiannis K, Mariolis A, Tountas $\mathrm{Y}$. Dietary patterns in relation to socio-economic and lifestyle characteristics among Greek adolescents: a multivariate analysis. Public Health Nutr. 2009;12(9):1366-72. https://doi. org/10.1017/S1368980008004060

56. Neumark-Sztainer D, Hannan PJ, Story M, Croll J, Perry C. Family meal patterns: associations with sociodemographic characteristics and improved dietary intake among adolescents. J Am Diet Assoc. 2003;103(3):317-22. https://doi.org/10.1053/jada.2003.50048

57. Stewart SD, Menning CL. Family structure, nonresident father involvement, and adolescent eating patterns. J Adolesc Health. 2009; 45(2):193-201. https://doi.org/10.1016/j.jadohealth.2009. 01.005 